\title{
Lattice position and thermal stability of diluted As in Ge
}

S. Decoster, ${ }^{1, \text { a) }}$ U. Wahl, ${ }^{2}$ S. Cottenier, ${ }^{3}$ J. G. Correia, ${ }^{2}$ T. Mendonça, ${ }^{4}$ L. M. Amorim, ${ }^{1}$

L. M. C. Pereira, ${ }^{1,2,4}$ and A. Vantomme ${ }^{1}$

1) Instituut voor Kern- en Stralingsfysica and INPAC, KULeuven, Celestijnenlaan 200D, 3001 Leuven, Belgium

${ }^{2)}$ Instituto Tecnológico e Nuclear, UFA, Estrada Nacional 10, apt. 21, 2686-953

Sacavém, Portugal

3) Center for Molecular Modeling and Department of Materials Science and Engineering, Ghent University, Technologiepark 903, 9052 Zwijnaarde, Belgium

4) IFIMUP and IN-Institute of Nanoscience and Nanotechnology, Departamento de Física, Faculdade de Ciências, Universidade do Porto, 4169-007 Porto, Portugal

(Dated: 23 January 2012)

We present a lattice location study of the $n$-type dopant arsenic after ion implantation into germanium. By means of electron emission channeling experiments, we have observed that the implanted As atoms substitute the Ge host atoms. However, in contrast to several implanted metal impurities in Ge, no significant fraction of As is found on interstitial sites. The substitutional As impurities are found to be thermally stable up to $600^{\circ} \mathrm{C}$. After $700^{\circ} \mathrm{C}$ annealing a strong reduction of emission channeling effects was observed, in full accordance with the expected diffusion-induced broadening of the As profile.

\footnotetext{
a)Electronic mail: stefan.decoster@fys.kuleuven.be
} 


\section{INTRODUCTION}

The most important advantage of Si over any other semiconducting material, i.e. its natural stable oxide $\mathrm{SiO}_{2}$, has reached its physical limits and is not able to follow the current down-scaling trend of semiconductor technology. Since $\mathrm{SiO}_{2}$ is being replaced by high- $\kappa$ materials, the quest for other semiconductor materials to replace Si is becoming more and more important. Because of its high carrier mobility and its compatibility with the well-studied Si-based technology, Ge is one of the promising candidates to replace $\mathrm{Si}$ as the active layer in semiconductor devices.

Both the diffusion and the activation behavior for p-type and n-type dopants in Ge have been investigated in detail. While p-type doping of Ge (with B) is quite promising, due to its low diffusion rate, n-type doping seems to be more problematic. Similar to P, As has shown transient enhanced diffusion, which limits the thermal budget that can be used during device processing ${ }^{1}$. The activation behavior of ion implanted As in Ge has been repeatedly studied and reported in literature as well. ${ }^{2-7}$ For low concentrations of ion implanted As in Ge (i.e. below $3 \times 10^{19} / \mathrm{cm}^{3}$ ), good to perfect activation has been observed after a typical annealing temperature of $500^{\circ} \mathrm{C} .^{2,3}$ However, when having higher As concentrations (up to a few atomic percent), the electrical activation level was found to be significantly smaller than the chemical concentration, with a maximum electrical activation of $\sim 4 \times 10^{19} / \mathrm{cm}^{3}{ }^{4-7}$

While it is widely accepted that the active fraction of group $\mathrm{V}$ dopants in Ge occupy the substitutional lattice site in group IV semiconductors, there is no direct information available in literature on the exact lattice location of As in Ge. The main reason for this can be found in the limitations of the commonly used experimental lattice location techniques. Because As and Ge have a comparable Z value, Rutherford backscattering spectrometry experiments in channeling geometry (RBS/C) are highly compromised, due to the overlap of their backscattering signals. Furthermore, fluorescent X-ray absorption techniques are difficult, because of the strong interference from the fluorescent X-rays from the Ge matrix with the fluorescent X-rays from the As dopants. Finally, techniques based on hyperfine interactions, such as Mössbauer spectroscopy, simply lack a suitable probe.

One of the very few experimental techniques, capable of providing detailed, accurate and direct information on the lattice location of As atoms in the Ge matrix, is electron emission channeling (EC). This technique has repeatedly proven its strength in determining the lattice 
site of a broad range of ion implanted impurities in $\mathrm{Ge}^{8-12}$ as well as in several other singlecrystalline materials. ${ }^{13-17}$ In particular, recent EC experiments in Ge have shown that this technique is capable of observing small fractions of impurities, both on substitutional and on interstitial sites, such as the bond-centered (BC) site $^{8-12}$ or the tetrahedral interstitial (T) site. $^{8}$

In this paper, we present an emission channeling study of the lattice location of ion implanted As in Ge, as well as of the thermal stability of the occupied lattice sites. We will show that the large majority of As atoms are located on an undisplaced substitutional site in Ge, which is in contrast to several metal impurities in Ge for which, besides substitutional sites, also large interstitial fractions were found. Furthermore, we will discuss experimental and theoretical indications that the As atoms do not occupy the so-called split-vacancy configuration in the As-monovacancy (As-V) complexes, unlike many other impurity-vacancy complexes in Ge, but instead prefer the full-vacancy configuration. Finally, we will also show that the EC experiment can be used to obtain information on the depth distribution of the diffused As atoms in Ge, which leads us to conclude that the As atoms also occupy the substitutional site after long-range diffusion.

\section{EXPERIMENTAL METHOD AND DETAILS}

When charged particles travel through the rows and planes of atoms in a single-crystalline material, they will be steered preferentially along or in between the atomic rows and planes, depending on their charge state. The electron emission channeling technique makes use of this effect by implanting a radioactive probe of the element under investigation in a single-crystalline matrix and then measuring the anisotropic emission of the charged decay particles along a selection of low-index crystalline directions with a two-dimensional energyand position-sensitive detector. ${ }^{18}$ These emission patterns are characteristic for the occupied lattice sites of the implanted probe atoms, and through measuring along several crystalline directions under the same conditions, it is possible to extract unique information on the lattice location of the probe atoms. Quantitative and qualitative information on the lattice location of the implanted impurities is obtained by fitting simulated patterns to the experimental data. The theoretical framework of these calculations is based on the so-called many beam formalism, and more information on these calculations and on the fitting pro- 
cedure can be found in Refs. 18, 19 and 20. Simulated patterns are calculated for a large number of high-symmetry sites, as well as for discrete displacements between these sites. In Fig. 1, a selection of high-symmetry sites in the (110)-plane of a diamond crystal lattice are presented, together with their corresponding electron emission patterns along the $\langle 211\rangle$ direction, for the experimental conditions used in this study. The significant differences between all of these emission patterns are a good indication of the strength of this technique to differentiate between a broad range of possible lattice sites. Finally, in order to obtain an accurate quantitative lattice location determination, the influence of electrons which are first backscattered in the sample or which scatter on the inside of the vacuum tubes of the experimental setup, needs to be taken into account. These electrons give rise to an isotropic background to the experimental patterns and therefore need to be extracted from the final results. This correction factor is obtained by performing GEANT $4^{21}$ simulations for the experimental vacuum setup, for each of the measured crystalline directions.
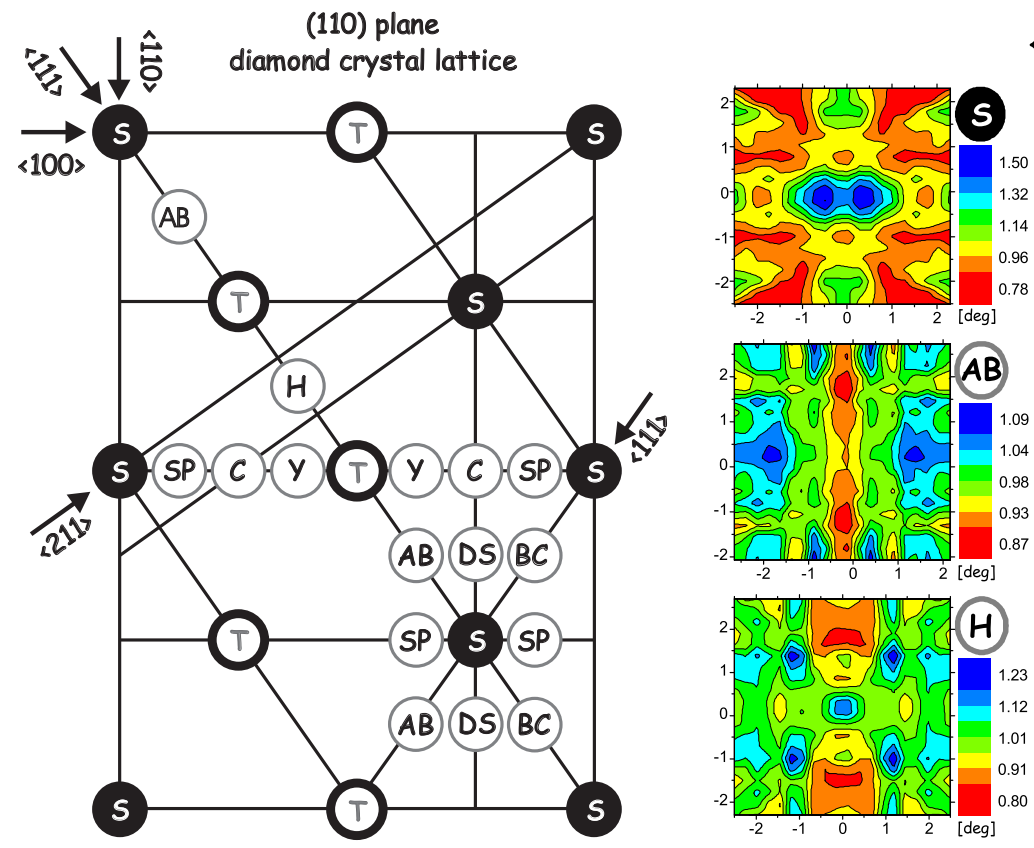

$<211>$ simulated patterns
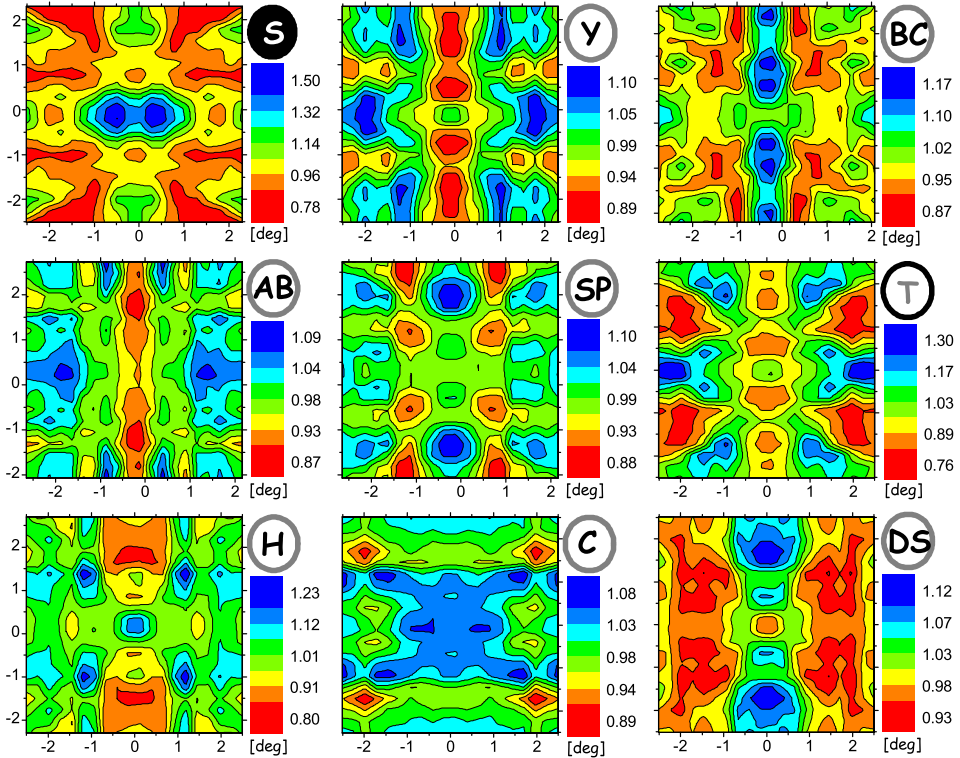

FIG. 1. (Color online) (left) High-symmetry sites on the (110) plane in a diamond crystal lattice: S (substitutional site), Y (ytterbium site), BC (bond-centered site), AB (anti-bonding site), SP (split site), $\mathrm{T}$ (tetrahedral interstitial site), $\mathrm{H}$ (hexagonal interstitial site), $\mathrm{C}\left(\mathrm{C}_{2 V}\right.$ site) and DS (displaced substitutional site); (right) simulated ${ }^{73}$ As emission patterns of these high-symmetry sites, as seen along the $\langle 211\rangle$ direction of Ge. 
In order to study the lattice location of $\mathrm{As}$ in $\mathrm{Ge}$, we have implanted the radioactive ${ }^{73}$ As isotope in undoped $\langle 111\rangle$-oriented bulk Ge. The room temperature implantations were performed at the on-line isotope separator (ISOLDE) facility at CERN, Geneva, with an energy of $60 \mathrm{keV}$ and a fluence of $1 \times 10^{13} / \mathrm{cm}^{2}$, resulting in a local maximum concentration of $2.5 \times 10^{18} / \mathrm{cm}^{3}$. The ${ }^{73}$ As isotope decays by electron capture to ${ }^{73} \mathrm{Ge}$ with a half life of $80.30 d$, emitting $42 \mathrm{keV}$ and $52 \mathrm{keV}$ conversion electrons. The electron emission patterns have been measured along the $\langle 111\rangle,\langle 100\rangle,\langle 110\rangle$ and $\langle 211\rangle$ direction. In situ in-vacuum $\left(<10^{-5}\right.$ mbar $) 10 \mathrm{~min}$ annealing steps were performed to study the thermal stability of the occupied lattice sites.

\section{RESULTS}

As a representative example for the rest of the experimental patterns, Fig. 2 (a-d) shows the measured 2-dimensional normalized conversion electron emission patterns from ion implanted ${ }^{73} \mathrm{As}$ in Ge after annealing at $400^{\circ} \mathrm{C}$, along the $\langle 111\rangle,\langle 100\rangle,\langle 110\rangle$ and $\langle 211\rangle$ direction, respectively. The colors in these patterns depict the normalized electron yield, from red (low yield) over green to blue (high yield). It is readily visible that all four patterns show an increased electron yield around the measured crystalline direction. This is characteristic for the channeling effect of substitutional probe atoms, since the negatively charged electrons are guided along the row of atoms (with a positively charged core) of this particular crystallographic direction. In order to obtain unambiguous results, all four patterns have been fitted simultaneously to a linear combination of simulated patterns for As on high-symmetry sites (see Fig. 1) with different dynamic displacements and discrete displacements between these sites.

Although previous EC results have indicated that several impurities in Ge not only occupy the substitutional site but also other high-symmetry sites, such as the BC site and the $\mathrm{T}$ site, ${ }^{8-12}$ an excellent fit to the presented data in Fig. 2 (a-d) which is consistent in all four directions, is obtained by assuming that all As atoms occupy an undisplaced substitutional site. Adding a second or third fraction to the substitutional fraction did not result in a significant fit improvement. The best fits to the experimental patterns in Fig. 2 (a-d) are presented in Fig. 2 (e-h), and clearly show a very good correspondence between experiment and fit. These fits correspond to a substitutional occupation of respectively $90 \%, 106 \%, 92 \%$ 

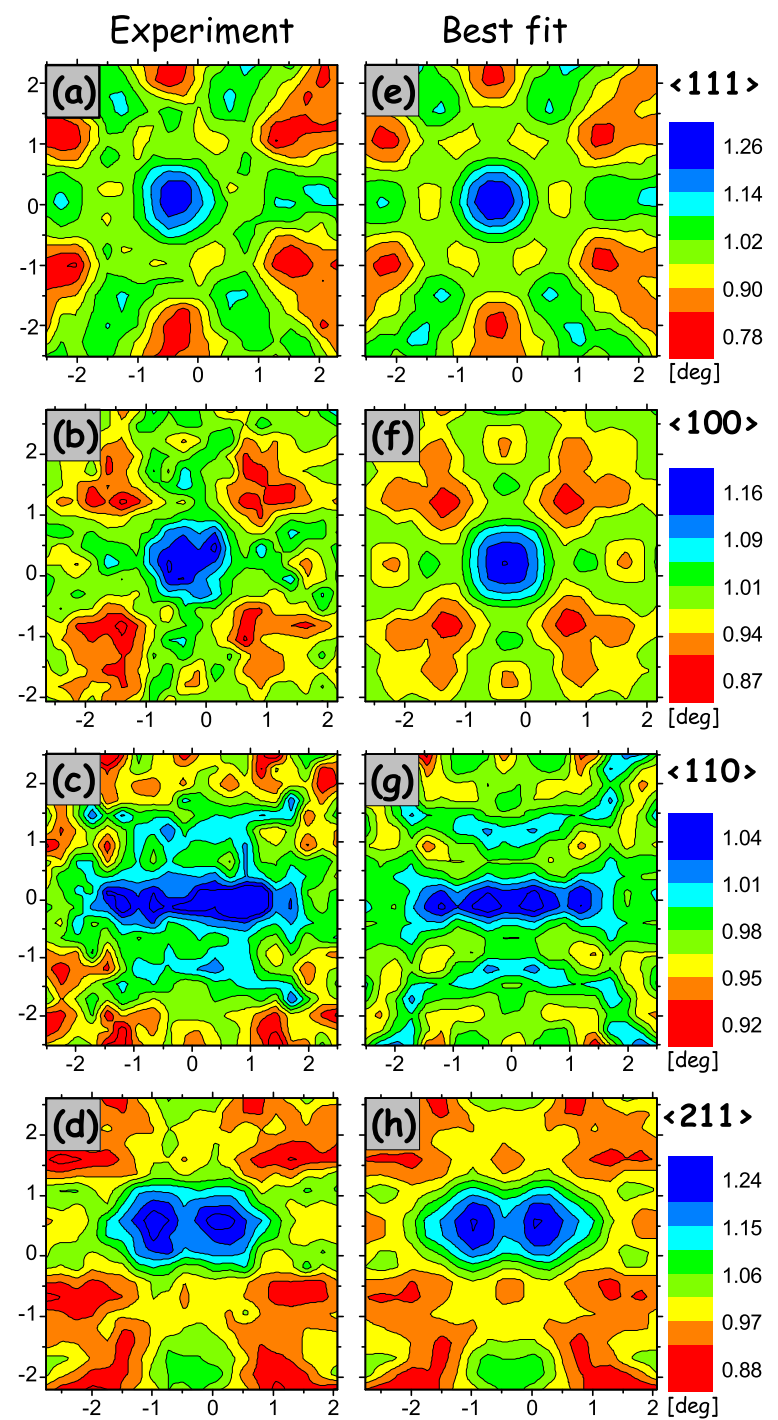

FIG. 2. (Color online) (a-d) Normalized conversion electron emission patterns from $60 \mathrm{keV}$ ion implanted ${ }^{73} \mathrm{As}$ in $\mathrm{Ge}$, after a $10 \mathrm{~min}$ in-vacuum annealing step at $400^{\circ} \mathrm{C}$, along the $\langle 111\rangle,\langle 100\rangle$, $\langle 110\rangle$ and $\langle 211\rangle$ direction, respectively; (e-h) the best fits to these experimental patterns, respectively, corresponding to $90 \%, 106 \%, 92 \%$ and $82 \%$ of the As atoms on the substitutional site with a root mean square displacement of $0.08 \AA$.

and $82 \%$, with a root mean square atomic displacement of $0.08 \AA$. Averaging over the four measured crystal directions, $93\left({ }_{-11}^{+13}\right) \%$ of the implanted As atoms are found to occupy an undisplaced substitutional site, after a $400^{\circ} \mathrm{C}$ annealing step in Ge.

This data analysis procedure has been applied to all the experimental patterns, and similar results have been obtained for the as-implanted state, as well as for the different 


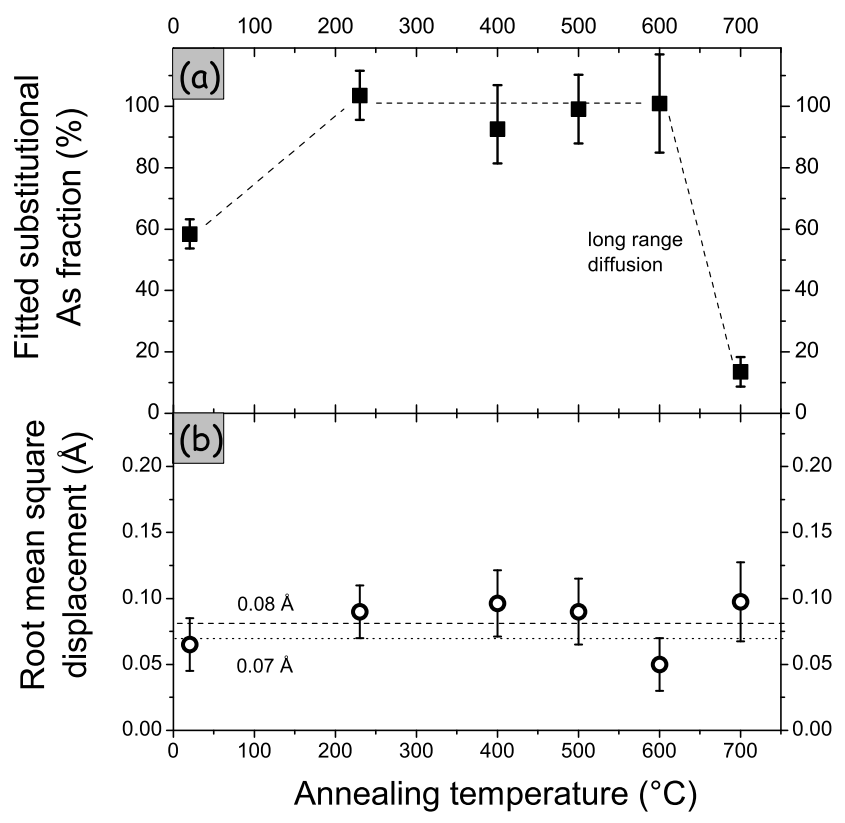

FIG. 3. (a) The fitted fraction of As atoms on the substitutional site in Ge as a function of annealing temperature, averaged for the four measured crystallographic directions. The error bars represent the spread in fitted fractions for the different crystalline directions. The dotted line is only to be used as a visual guideline; (b) The root mean square displacement of the substitutional As atoms, as obtained from averaging the best fits of the four measured crystallographic directions for each annealing temperature. The dashed line represents the value $(0.08 \AA)$ used for the fitted substitutional fractions in (a), as well as for the best fit patterns in Fig. 2 (e-h). The dotted line $(0.07 \AA)$ represents the expected thermal root mean square displacement value at room temperature.

annealing steps up to $700^{\circ} \mathrm{C}$, i.e. the As atoms only occupy an undisplaced substitutional site in Ge. An overview of these results is presented in Fig. 3 (a), where the fraction of the As atoms that are found on the undisplaced substitutional site is plotted as a function of the annealing temperature. These values are obtained by averaging the fit results to the emission patterns around the four measured crystallographic directions, and the error bars represent the spread of the four fitted fractions.

In order to study the displacement of the As atoms from perfect substitutional sites, both a static displacement as well as a dynamic displacement can be applied to the simulated patterns used in the fitting procedure. While a static displacement - i.e. assuming that all As atoms occupy a site which is displaced from the substitutional site in a particular direction 
- did not result in an improved fit, we have also varied the root mean square displacement (dynamical displacement) of the substitutional As impurities. The dynamical displacement values resulting in the best fit to the experimental patterns, when leaving this parameter free in the fitting procedure, are shown in Fig. 3 (b). From this analysis, an average dynamical displacement of $0.08 \AA$ (dashed line) is found, and this value has been fixed to obtain the fit results shown in Fig. 3 (a). This value is close to the expected thermal vibration (root mean square displacement) of the As atoms in Ge at room temperature, i.e. $0.07 \AA$ (dotted line in Fig. 3 (b)). Therefore, we can conclude that the majority of the As atoms are located on an undisplaced substitutional site.

\section{DISCUSSION}

\section{A. Substitutional fraction}

As can be seen in Fig. 3 (a), close to $100 \%$ of the implanted As atoms occupy the undisplaced substitutional site for the annealing steps from $230^{\circ} \mathrm{C}$ to $600^{\circ} \mathrm{C}$. This is in good agreement with electrical activation studies, where high activation levels were observed for a low concentration of implanted As atoms. ${ }^{2,3}$

However, there are two features in Fig. 3 (a) that need more explanation. First of all, there is a clear reduction in the fitted substitutional fraction after annealing at $700^{\circ} \mathrm{C}$. This reduction can be attributed to diffusion, and will be explained in detail below. Secondly, the fitted substitutional fraction in the as-implanted state is significantly smaller than after the first annealing step at $230^{\circ} \mathrm{C}$. This effect can be related to implantation-induced defects in the Ge crystal lattice. Because of these implantation-induced defects, some of the implanted As dopants will be embedded in a defective crystalline region, which will reduce the channeling effect and render the electron emission more isotropic. Moreover, some of the conversion electrons, emitted from As atoms located in a defect-free crystalline region, will travel through damaged crystalline regions, which increases their dechanneling probability, resulting in a weaker channeling effect and, consequently, in a more isotropic emission pattern. Therefore, the lower fitted substitutional fraction in the as-implanted state does not necessarily mean that less As atoms occupy the substitutional site, but is most likely a sign of implantation-induced defects in the Ge lattice. For similar reasons, the increase in fitted 
substitutional fraction after annealing at $230^{\circ} \mathrm{C}$ can be attributed to the local recovery of the defective Ge matrix. This effect has also been observed in other EC experiments in $\mathrm{Ge}^{8-11,22}$ as well as in $\mathrm{Si}^{13}$

\section{B. As-V complexes}

Although there is no direct way from this emission channeling experiment to determine whether As-V complexes are present in the studied sample, there are several reasons to expect their presence. First of all, it is known that ion implantation creates a excessive number of vacancies, which are mobile in Ge at room temperature. ${ }^{23}$ A significant fraction of these vacancies will recombine with self-interstitials or form multivacancy complexes, but it can also be expected that a smaller fraction will be trapped by the As impurities. Secondly, substitutional As impurities in Ge, as group V elements in a group IV semiconductor, are positively charged donors which electrostatically attract the negative vacancies and are therefore expected to be effective vacancy traps in Ge. ${ }^{24,25}$ Finally, in a large number of EC lattice location studies for implanted impurities in $\mathrm{Ge}^{8-12,22}$ as well as in $\mathrm{Si},{ }^{14,15,26}$ there are strong indications that impurity-vacancy complexes are present. These reasons indicate that it is very likely that As-V complexes are present in the investigated sample, and the consequences of their possible presence will be discussed here.

From the fit results of this EC experiment, there is no sign of interstitial As atoms after ion implantation in Ge. ${ }^{27}$ This finding is in clear contrast to what was found from earlier EC experiments for a range of other impurities $\left(\mathrm{Mn}^{12}, \mathrm{Fe}, \mathrm{Cu}\right.$ and $\mathrm{Ag}^{9}, \mathrm{In}^{10}, \mathrm{Sn}^{11}$ and $\left.\mathrm{Er}^{8}\right)$ in $\mathrm{Ge}$, where a significant fraction of these impurities was found on the bond-centered site, i.e. a site in the center of two nearest neighbor substitutional sites. This behavior was attributed to the capture of vacancies by substitutional impurity atoms. Corroborated by density functional theory (DFT) calculations, ${ }^{9,11,12}$ it was concluded, that the impurity-vacancy complex in the so-called full-vacancy configuration - i.e. with the impurity on the substitutional site and a vacancy as nearest neighbor - is unstable and results in a large force on the impurity along the $\langle 111\rangle$ direction. The impurity is then pushed towards the vacant lattice position and a stable configuration is reached when the impurity occupies the bond-centered site with two vacant lattice positions on each neighboring substitutional site, i.e. the so-called splitvacancy configuration. A visual representation of these two impurity-vacancy configurations 
can be found in Ref. 11.

Since we have not seen any sign of As on the BC site, and since we do expect As-V complexes to be present, this leads us to believe that the As-V complexes prefer the fullvacancy configuration. Moreover, since no large displacements are found (see Fig. 3 (b)), it can be expected that the As atoms in the As-V complexes occupy a lattice site very close to the substitutional site. In order to verify this statement, we have performed complementary DFT calculations. The calculations were done by the APW+lo method ${ }^{28}$ within $\mathrm{DFT},{ }^{29}$ as implemented in the WIEN2K code. ${ }^{30,31}$ More details of these calculations can be found in Refs. 9 and 11. From these calculations, it was found that, in contrast to many other impurity-vacancy complexes in Ge, the split-vacancy configuration of the As- $\mathrm{V}$ complex is not stable and relaxes to the stable full-vacancy configuration. The As impurity in the fullvacancy configuration of the As-V complex was calculated to be slightly displaced (by $0.28 \AA$ ) from the ideal substitutional site. These findings are in agreement with literature, where the full-vacancy configuration for the As-V complex was calculated by different implementations of DFT theory to be energetically more favorable than the split-vacancy configuration, and where displacements of $0.19-0.24 \AA$ from the perfect $\mathrm{S}$ site were obtained. ${ }^{32-35}$ Furthermore, all these calculations are in agreement with the experimental results obtained here, ${ }^{36}$ and although we have no unambiguous experimental proof that $\mathrm{As}-\mathrm{V}$ complexes are present in this study, we have strong experimental and theoretical indications that As- $\mathrm{V}$ complexes prefer the full-vacancy configuration over the split-vacancy configuration, in contrast to several other impurities (Mn, Fe, Cu, Ag, In, Sn and Er) in Ge.

It must be noted that the observation of an As- $\mathrm{V}$ complex has been reported in literature. From deep level transient spectroscopy, an electron trap was attributed to an As-V complex after high energy $\gamma$-irradiation of As-doped Ge, and this trap was found to disappear between $100^{\circ} \mathrm{C}$ and $150^{\circ} \mathrm{C} .{ }^{37}$ However, from the EC data analysis presented here, we can only conclude that the As atoms have the same lattice location behavior in the as-implanted state and after the first annealing step at $230^{\circ} \mathrm{C}$, only with different fractions. Assuming that the observed electron trap is indeed related to an As-V complex, this could mean (1) that the As atoms in the As-V complexes occupy a perfect substitutional site, which makes them undistinguishable from As atoms without a nearest neighbor vacancy, or (2) that there are very limited or no As- $\mathrm{V}$ complexes present in the investigated sample. 


\section{As diffusion}

As mentioned above, the drastic reduction in the fitted substitutional fraction after annealing at $700^{\circ} \mathrm{C}$ for $10 \mathrm{~min}$ in vacuum, seems not to be related to a change in the lattice site behavior of the implanted As atoms. Instead, it can be attributed to diffusion, which is in agreement with the fact that As impurities are mobile in Ge at this temperature. ${ }^{2,5,38-43}$ The reduced fitted substitutional fraction can be explained as follows. When an emitted electron channels through a single-crystalline material, there is a probability that the electron will be dechanneled, which increases with longer travel distance inside the material. Therefore, an important input parameter for the simulated emission patterns, is the depth profile of the probe atoms. According to the experimental conditions in this study, we have mimicked the depth profile of the $60 \mathrm{keV}{ }^{73}$ As impurities in Ge with a Gaussian distribution around 312 $\AA$ and a standard deviation of $155 \AA$. However, this depth profile is not accurate anymore when diffusion comes into play and can lead to an underestimation of the fitted fractions, as can be seen in Fig. 3 (a).

From the qualitative analysis of the experimental patterns after annealing at $700^{\circ} \mathrm{C}$, it can be concluded that the As atoms do not occupy any other high-symmetry sites. As can be seen in Fig. 3 (b), the dynamic displacement value is still comparable to the expected thermal displacement value, indicating that the As atoms also do not occupy a displaced substitutional site. Therefore, in the following analysis, we will assume that all of the remaining As atoms occupy an undisplaced substitutional site. From this assumption, it is possible to estimate the diffusion width of As atoms after annealing at $700^{\circ} \mathrm{C}$.

In order to do this, we have simulated the ${ }^{73}$ As emission patterns around the $\langle 111\rangle$ direction for a range of different Gaussian depth profiles around the projected range of 312 $\AA .{ }^{44}$ The standard deviation or width $(\sigma)$ of these Gaussian depth profiles has been varied from $200 \AA$ to $10000 \AA$. Three such simulations are shown in Figs. 4 (a-c), for a Gaussian distribution with $\sigma$ equal to $200 \AA, 2500 \AA$ and $10000 \AA$, respectively. From these patterns, it is clear that the anisotropy drastically decreases when the As atoms are more spread throughout the bulk Ge matrix.

The results from fitting the $\langle 111\rangle$ experimental pattern after the $700^{\circ} \mathrm{C}$ annealing step to the simulations with different depth profiles are shown in Fig. 4 (d), where the fitted substitutional fraction is plotted as a function of the width of the Gaussian depth profile 


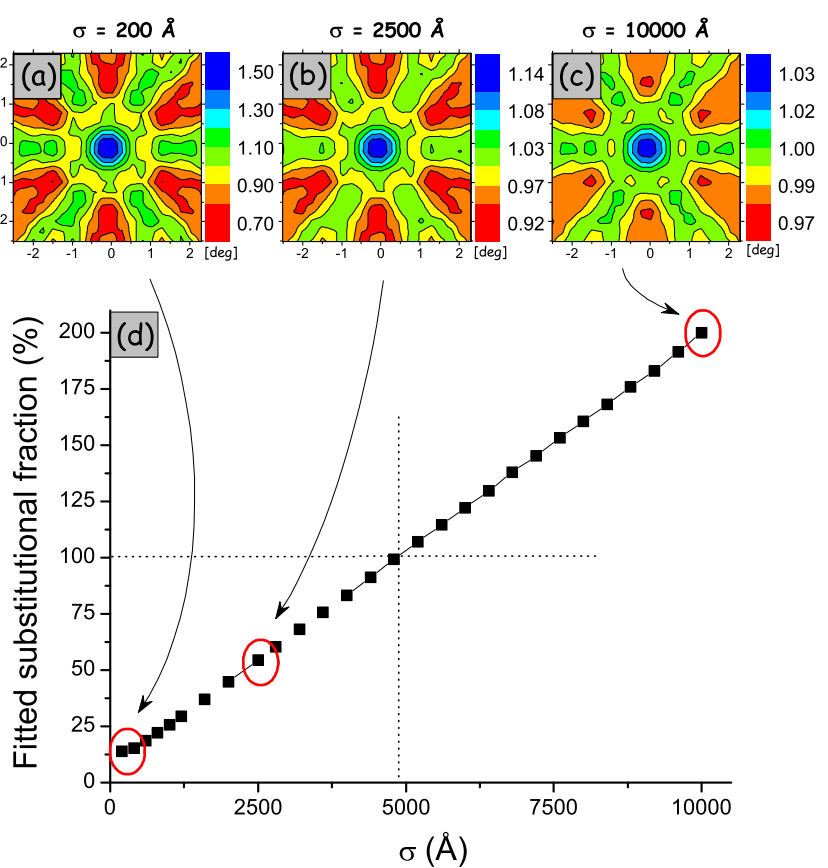

FIG. 4. (Color online) (a)-(c) Simulated electron emission patterns around the $\langle 111\rangle$ direction for a Gaussian depth distribution of ${ }^{73}$ As impurities in Ge around $312 \AA$ with a standard deviation of $\sigma=200 \AA, 2500 \AA$ and $10000 \AA$, respectively; (d) The fitted substitutional fraction to the experimental pattern around the $\langle 111\rangle$ direction after annealing at $700^{\circ} \mathrm{C}$, as a function of the width of the simulated Gaussian depth distribution of As impurities.

of the As atoms. By assuming that all of the remaining As atoms occupy an undisplaced substitutional site, we can conclude that the depth profile of the diffused As atoms can be represented by a Gaussian distribution with $\sigma=4900 \AA$. This value will now be compared to the available data from earlier diffusion studies in literature.

In Tab. I, we present an overview of the pre-exponential factors $D_{0}$ and the activation energies $E_{a}$, as extracted from different As diffusion experiments in Ge from Refs. 38-41, and 43. We also included the calculated activation energies for singly negatively charged and neutral Ge vacancies, as obtained from recent DFT calculations, ${ }^{35}$ which are in good agreement with the most recent experimental values. ${ }^{43}$ From all of these published experimental values, we have calculated the diffusion coefficient $D$ and the diffusion length $l$ for a $600 \mathrm{~s}$ annealing step at a temperature of $700^{\circ} \mathrm{C}$, from $D=D_{0} \exp \left(-E_{a} / k_{B} T\right)$ (where $k_{B}$ is Boltzmann's constant and $T$ is the absolute temperature) and $l=\sqrt{2 D t}$, respectively. These values can be found in Tab. I, and it can be concluded that the extracted width of the 


\begin{tabular}{|c|c|c|c|c|}
\hline $\begin{array}{l}D_{0} \\
\left(\mathrm{~cm}^{2} / \mathrm{s}\right)\end{array}$ & $\begin{array}{l}E_{\mathrm{a}} \\
(\mathrm{eV})\end{array}$ & $\begin{array}{l}D_{700^{\circ} \mathrm{C}} \\
\left(\mathrm{cm}^{2} / \mathrm{s}\right)\end{array}$ & $\begin{array}{l}l_{600 s} \\
\left(\times 10^{3} \AA\right)\end{array}$ & Ref. \\
\hline $1.66 \times 10^{-2}$ & 1.83 & $5.51 \times 10^{-12}$ & 8130 & $38^{[\mathrm{a}]}$ \\
\hline $9.33 \times 10^{-5}$ & 1.57 & $6.88 \times 10^{-13}$ & 2870 & $38^{[\mathrm{b}]}$ \\
\hline $2.02 \times 10^{6}$ & 3.4 & $5.51 \times 10^{-12}$ & 7400 & $39^{[\mathrm{a}]}$ \\
\hline $1.89 \times 10^{2}$ & 2.9 & $6.88 \times 10^{-13}$ & 1420 & $39^{[\mathrm{b}]}$ \\
\hline $5.8 \times 10^{0}$ & 2.42 & $9.21 \times 10^{-12}$ & 4380 & 40 \\
\hline $1.45 \times 10^{6}$ & 3.32 & $9.21 \times 10^{-12}$ & 1050 & 41 \\
\hline \multirow[t]{3}{*}{$3.20 \times 10^{1}$} & 2.71 & $2.94 \times 10^{-13}$ & 1880 & 43 \\
\hline & 2.67 & & & $35^{[c]}$ \\
\hline & 2.56 & & & $35^{[\mathrm{a}]}$ \\
\hline
\end{tabular}

TABLE I. The experimental pre-exponential factor $D_{0}$ and the activation energy $E_{\mathrm{a}}$ from Refs. $38-$ 41 , and 43 as well as the calculated diffusion coefficient $D_{700^{\circ} \mathrm{C}}$ and diffusion length $l_{600 s}$ for a 600 s annealing step at a temperature of $700^{\circ} \mathrm{C}$, using the published $D_{0}$ and $E_{\text {a }}$ values; the activation energy from density functional theory calculations from Ref. 35 for singly negatively charged and neutral Ge vacancies.

[a] [b] [c] The quoted values refer to As diffusion via neutral [a], doubly negatively charged [b] and singly negatively charged [c] Ge vacancies, respectively.

Gaussian depth profile from the EC experiments after a 10 min $700^{\circ} \mathrm{C}$ annealing step $(4900$ $\AA)$ agrees well with the diffusion lengths deduced from the experimental diffusion studies from literature, which range from roughly $1000 \AA$ to $8000 \AA .{ }^{45}$ This, in turn, provides a good indication of the validity of the assumptions used to extract the depth profile, i.e. that the majority of the As atoms still occupy the substitutional site, even after long-range diffusion in $\mathrm{Ge}$, whereas the decrease of the observed channeling effect is merely diffusion-related.

\section{CONCLUSION}

We have performed an emission channeling study of the lattice location of ion implanted As atoms in Ge. It was shown in a direct way that this donor occupies an undisplaced substitutional site in the diamond lattice structure of Ge. Contrary to earlier EC experiments, 
where several impurities were found to occupy interstitial sites as well as substitutional sites, ${ }^{8-12}$ no As atoms were found on any interstitial site or on a displaced substitutional site. These results strongly indicate that As-V defect complexes, which are expected to be present in this study, do not occupy the split-vacancy configuration but prefer the full-vacancy configuration, i.e. with the As atom and a vacancy on nearest neighbor substitutional sites. This has been corroborated by DFT calculations, and was found to be in agreement with other theoretical studies. ${ }^{32-35}$ Furthermore, we have shown that a $700^{\circ} \mathrm{C}$ annealing step leads to the in-diffusion of As atoms, and that the diffusion width of the As impurities, estimated by making some reasonable assumptions, is in good agreement with literature values derived from detailed diffusion studies.

\section{ACKNOWLEDGMENTS}

We greatly acknowledge the support from the Research Foundation, Flanders (FWO G.0501.07, G.0636.08), the K.U.Leuven projects GOA/2009/006 and INPAC EF/2005/005, the IUAP P6/42 program, the EURONS project (RII3-CT-2004-506065) and the Portuguese Foundation for Science and Technology (Grant Nos. CERN/FP/116320/2010 and SFRH/BD/35761/2007). S. C. acknowledges support from OCAS NV by an OCAS-endowed industrial chair at Ghent University.

\section{REFERENCES}

${ }^{1}$ C. Claeys and E. Simoen, Germanium-based technologies: From materials to devices, Elsevier, Amsterdam (2007).

${ }^{2}$ A. Axmann, M. Schulz, and C. R. Fritzsche, Appl. Phys. 12, 173 (1977).

${ }^{3}$ S. V. Hattangady, G. G. Fountain, E. H. Nicollian, and R. J. Markunas, J. Appl. Phys. 63, 68 (1988).

${ }^{4}$ M. Koike, Y. Kamata, T. Ino, D. Hagishima, K. Tatsumura, M. Koyama, and A. Nishiyama, J. Appl. Phys. 104, 023523 (2008).

${ }^{5}$ S. Koffel, R. J. Kaiser, A. J. Bauer, B. Amon, P. Pichler, J. Lorenz, L. Frey, P. Scheiblin, V. Mazzocchi, J. P. Barnes, and A. Claverie, Microelec. Eng. 88, 458 (2011). 
${ }^{6}$ C. O. Chui, L. Kulig, J. Moran, W. Tsai, and K. C. Saraswat, Appl. Phys. Lett. 87, 091909 (2005).

${ }^{7}$ A. Satta, E. Simoen, T. Janssens, T. Clarysse, B. De Jaeger, A. Benedetti, I. Hoflijk, B. Brijs, M. Meuris, and W. Vandervorst, J. Electrochem. Soc. 153, G229 (2006).

${ }^{8}$ S. Decoster, B. De Vries, U. Wahl, J. G. Correia, and A. Vantomme, Appl. Phys. Lett. 93, 141907 (2008).

${ }^{9}$ S. Decoster, S. Cottenier, B. De Vries, H. Emmerich, U. Wahl, J. G. Correia, and A. Vantomme, Phys. Rev. Lett. 102, 065502 (2009).

${ }^{10}$ S. Decoster, B. De Vries, U. Wahl, J. G. Correia, and A. Vantomme, J. Appl. Phys. 105, 083522 (2009).

${ }^{11}$ S. Decoster, S. Cottenier, U. Wahl, J. G. Correia, and A. Vantomme, Phys. Rev. B 81, 155204 (2010).

${ }^{12}$ S. Decoster, S. Cottenier, U. Wahl, J. G. Correia, L. M. C. Pereira, C. Lacasta, M. R. Da Silva, and A. Vantomme, Appl. Phys. Lett. 97, 151914 (2010).

${ }^{13}$ U. Wahl, A. Vantomme, J. De Wachter, R. Moons, G. Langouche, J. G. Marques, J. G. Correia, and the ISOLDE Collaboration, Phys. Rev. Lett. 79, 2069 (1997).

${ }^{14}$ U. Wahl, A. Vantomme, G. Langouche, J. G. Correia, and the ISOLDE Collaboration, Phys. Rev. Lett. 84, 1495 (2000).

${ }^{15}$ U. Wahl, J. G. Correia, E. Rita, J. P. Araújo, J. C. Soares, and the ISOLDE Collaboration, Phys. Rev. B 72, 014115 (2005).

${ }^{16}$ U. Wahl, J. G. Correia, T. Mendonça, and S. Decoster, Appl. Phys. Lett. 94, 261901 (2009).

${ }^{17}$ L. M. C. Pereira, U. Wahl, S. Decoster, J. G. Correia, M. R. da Silva, A. Vantomme, and J. P. Araújo, Appl. Phys. Lett. 98, 201905 (2011).

${ }^{18}$ U. Wahl, J. G. Correia, A. Czermak, S. G. Jahn, P. Jalocha, J. G. Marques, A. Rudge, F. Schopper, J. C. Soares, A. Vantomme, P. Weilhammer, and the ISOLDE Collaboration, Nucl. Instrum. Methods Phys. Res. A 524, 245 (2004).

${ }^{19}$ H. Hofsäss and G. Lindner, Phys. Rep. 201, 121 (1991).

${ }^{20}$ U. Wahl, Hyperf. Int. 129, 349 (2000).

${ }^{21}$ S. Agostinelli et al., Nucl. Instrum. Methods A 506, 250 (2003).

${ }^{22}$ U. Wahl, J. G. Correia, J. C. Soares, and the ISOLDE Collaboration, Physica B 340, 799 (2003). 
${ }^{23}$ H. Haesslein, R. Sielemann, and C. Zistl, Phys. Rev. Lett. 80, 2626 (1998).

${ }^{24}$ S. Brotzmann, H. Bracht, J. L. Hansen, A. N. Larsen, E. Simoen, E. E. Haller, J. S. Christensen, and P. Werner, Phys. Rev. B 77, 235207 (2008).

${ }^{25}$ M. Naganawa, Y. Shimizu, M. Uematsu, K. M. Itoh, K. Sawano, Y. Shiraki, and E. E. Haller, Appl. Phys. Lett. 93, 191905 (2008).

${ }^{26}$ U. Wahl, A. Vantomme, G. Langouche, J. P. Araújo, L. Peralta, and J. G. Correia, Appl. Phys. Lett. 77, 2142 (2000).

${ }^{27}$ Due to the statistical limits of the presented data, a very small fraction $(<3$ percent) of interstitial As atoms cannot be excluded.

${ }^{28}$ E. Sjöstedt, L. Nordström, and D. J. Singh, Solid State Commun. 114, 15 (2000).

${ }^{29}$ P. Hohenberg and W. Kohn, Phys. Rev. 136, 864 (1964).

${ }^{30}$ S. Cottenier, (Instituut voor Kern- en Stralingsfysica, KULeuven, Belgium) (2002), ISBN 90-807215-1-4 (freely available from http://www.wien2k.at/reg_user/textbooks).

${ }^{31}$ P. Blaha, K. Schwarz, G. Madsen, D. Kvasnicka, and J. Luitz, (Karlheinz Schwarz, Techn. Universität Wien, Austria) (1999), ISBN 3-9501031-1-2.

${ }^{32}$ H. Höhler, N. Atodiresei, K. Schroeder, R. Zeller, and P. H. Dederichs, Phys. Rev. B 71, $035212(2005)$.

${ }^{33}$ J. Coutinho, S. Öberg, V. J. B. Torres, M. Barroso, R. Jones, and P. R. Briddon, Phys. Rev. B 73, 235213 (2006).

${ }^{34}$ A. Chroneos, R. W. Grimes, B. P. Uberuaga, S. Brotzmann, and H. Bracht, Appl. Phys. Lett. 91, 192106 (2007).

${ }^{35}$ H. Tahini, A. Chroneos, R. W. Grimes, U. Schwingenschlögl, and H. Bracht, Appl. Phys. Lett. 99, 072112 (2011).

${ }^{36}$ Although the emission channeling technique is capable of observing displacements of the order of $0.2 \AA$, it is not possible to accurately determine the fraction of slightly displaced probe atoms when a large undisplaced substitutional fraction is believed to be present, as is the case in this study.

${ }^{37}$ V. P. Markevich, I. D. Hawkins, A. R. Peaker, K. V. Emtsev, V. V. Emtsev, V. V. Litvinov, L. I. Murin, and L. Dobaczewski, Phys. Rev. B 70, 235213 (2004).

${ }^{38}$ E. Vainonen-Ahlgren, T. Ahlgren, J. Likonen, S. Lehto, J. Keinonen, W. Li, and J. Haapamaa, Appl. Phys. Lett. 77, 690 (2000). 
${ }^{39}$ T. Ahlgren, J. Likonen, S. Lehto, E. Vainonen-Ahlgren, and J. Keinonen, AIP Conf. Proc. 576, 887 (2001).

${ }^{40}$ P. Laitinen, I. Riihimäki, J. Räisänen, and the ISOLDE Collaboration, Phys. Rev. B 68, 155209 (2003).

${ }^{41}$ C. O. Chui, K. Gopalakrishnan, P. B. Griffin, J. D. Plummer, and K. C. Saraswat, Appl. Phys. Lett. 83, 3275 (2003).

${ }^{42}$ H. Bracht and S. Brotzmann, Mat. Sci. Semicond. Proc. 9, 471 (2006).

${ }^{43}$ S. Brotzmann and H. Bracht, J. Appl. Phys. 103, 033508 (2008).

${ }^{44}$ In order to choose the most appropriate type of depth profile, one needs to know the role of the Ge surface. While we can exclude any significant out-diffusion from the measured count rate during the experiments, it is not known whether the surface acts more as a mirror, reflecting the As atoms back into the bulk Ge, or instead acts more as a trap for the diffusing As atoms. While a Gaussian profile is a good approximation of the depth profile for a mirror-like surface, it must be noted that the calculated depth profile in this study will be overestimated in the case of significant trapping of As atoms at the Ge surface.

${ }^{45}$ The difference between the diffusion length obtained in this study, and the values deduced from published experimental diffusion studies, are most likely related to the different dopant concentrations, and to the different experimental conditions in general. 